\title{
Development of a Scale to Measure Memorable Tourism Experiences
}

\author{
Jong-Hyeong $\mathrm{Kim}^{1}$
}

Received: 14/09/2010

\begin{abstract}
1 Post-doctoral fellow, Department of Tourism and Marketing, Haskayne School of Business, University of Calgary; Phone: (403) 813-8817, e-mail: jonkim@ucalgary.ca
\end{abstract}

Supervisor: Dr. Bryan P. McCormick

Institution awarding the Ph. D. Degree: Indiana University

Date of defence: 8 June, 2009 (Final approval: 12 Oct, 2009)

(c) 2010 International University College. All rights reserved

Citation: Kim, J.H. (2010) Development of a Scale to Measure Memorable Tourism Experiences. Doctoral dissertation summary. European Journal of Tourism Research 3(2), pp. 123-126

\section{Goal and objectives of the dissertation Goal}

The goal of this study was to develop a valid and reliable instrument that would offer tourism managers and planners a useful tool in understanding and planning for memorable tourism experiences (MTE).

\section{Objectives}

Specifically, this study sought to answer the following research questions:

1. Does the MTE scale developed in this study yield an appropriate level of reliability and validity?

2. Are there relationships among the constructs in the proposed model?

3. How well does the construct model fit the data when tested using the analysis technique of structural equation model?

\section{Methodology}

Participants and Procedures

An empirical evaluation of the developed instrument was undertaken using data obtained from U.S. college students at a large Midwestern university. The students were enrolled in 12 classes spread across different academic majors. The participants were first asked to recall their MTE and to evaluate all 85 items on a seven-point Likert-type scale, on which 1 represented I have not experienced at all and 7 represented I have experienced very much. A total of 730 survey questionnaires were handed out and 536 copies were returned, representing an overall response rate of $73.4 \%$.

Development and validation of research instrument

In refining the scale, both exploratory (EFA) and confirmatory factor analyses (CFA) as well as reliability tests were conducted. After the number of factors was identified and the problematic items were removed based on the results of exploratory factor analysis, a confirmatory factor analysis was conducted using the covariance matrmatrix examine whether the scale items were structurally 
consistent with the embedded theory or with any a priori logic of scale design. Before the CFA, the sample was randomly split into two 250-case subsamples using SPSS routine for random case selection. One sample was used for calibration and the other for validation.

\section{Structural Equation Modeling}

After the convergent and discriminant validity are identified, a further examination of the causal relationships between the derived constructs and other constructs or variables of interest was performed to see if the derived constructs behave in the way stipulated by the underlying tenets guiding the MTE scale development. The resulting data further provides critical indication of construct validity. Researchers regarded this test as a process to assess nomological validity of construct (Anderson \& Gerbing, 1988; Campbell, 1960; Chen \& Hsu, 2001).

\section{Results}

\section{Purification of Measurement Scale}

In assessing the quality of an item, the itemto-total correlations were examined. This is a commonly accepted procedure in developing a scale (Choi \& Sirakaya, 2005). In order to purify the scale, items that were poorly correlated $(r<0.4)$ with the total score were eliminated. The reliability of the resulting 24item scale was 0.91 . Following the widely recognized rule of thumb to use a reliability level of 0.7 , this result indicated that the 24item MTE scale is highly reliable.

\section{Exploratory Factor Analysis}

EFA was followed by a rotation with both orthogonal (VARIMAX) and oblique (OBLIMIN) methods. Results from two different methods showed similar structures with the same value of explained variances. The results obtained from principal components analysis with an oblique rotation method was used for the data analysis in order to permit the obtained components to correlate and, therefore, followed the assumption that MTE components are related yet distinct from each other (Lee \& Comrey 1979). In deciding to retain an item, both factor loading $(r>0.4)$ and the communality $(r>0.5)$ of each item were examined. Employing a combination of Cattell (1966) scree test and theoretical basis of the scale (i.e., eigenvalues greater than 1), the EFA of the MTE scale extracted seven factors accounting for $74.63 \%$ of the total variance. The seven factors are hedonism, refreshment, local culture, meaningfulness, knowledge, involvement, and novelty.

\section{Confirmatory Factor Analysis}

The first step in interpreting the results of CFA is assessing overall fit of the model. The overall model fit was evaluated statistically by the chi-square test and heuristically using a number of goodness-of-fit statistics, such as Bentler's (1992) comparative fit index (CFI), Bentler and Bonett's (1980) non-normed fit index (NNFI), the incremental fit index (IFI), and the root mean square error of approximation (RMSEA). Results of the CFA showed that the measurement model fit the data very well. The ratio of the $x^{2}$ to the degrees of freedom $\left(x^{2} / d f=1.66\right)$ and other commonly used goodness-of-fit indices (CFI= $0.98, \mathrm{NNFI}=0.97, \mathrm{IFI}=0.98$, and $\mathrm{RMSEA}=$ $0.05)$ were in line with the established criteria (CFI above 0.90, NNFI above 0.95, and RMSEA below 0.08; Bentler, 1992; Hu \& Bentler, 1999; Jöreskog \& Sörbom, 1996). The reliability of the measure was then assessed by examining Cronbach's alphas and calculating composite reliability estimates. The Cronbach's alphas of all constructs $(0.80-0.87)$ indicated an acceptable internal consistency across the items in the constructs (Litwin, 1995). Moreover, the composite reliability estimates, ranging from 0.81 to 0.90 , indicated adequate internal consistency for multiple indicators for each construct in the model (i.e., composite reliabilities $>0.7$ ).

The next step was to assess the convergent validity and discriminant validity. Convergent validity was evaluated by checking the values of all the factor loadings for individual items and the average variance extracted. The results showed that the estimated values of AVE of all eight constructs were found to be greater than the unexplained variances (i.e., AVE > 0.5) and all of the factor loadings for 
the individual items were significant $(>0.5)$. Thus, this confirmed the convergent validity of the latent constructs. The discriminant validity of the measurement was investigated following Fornell and Larcker's (1981) suggestion. According to them, the AVE must exceed the corresponding correlation estimate between the two factors. In the current study, the shared variance between any two constructs was not greater than the AVE of the construct.

\section{Model Assessment in the Validation Sample}

The indicators exhibited statistically significant standardized factor loadings, composite reliabilities, and AVE. The AVE of each construct and the inter-correlations provided support for construct validity and discriminant validity. Moreover, the CFA model for the validation sample showed a good fit $\left(X^{2}(\mathrm{df}=221)\right.$ $=340.55(p<0.001), C F I=0.98$, NNFI= 0.98, $|F|=0.98$, and RMSEA $=0.05)$. These results are evidence that the proposed model of MTE is viable and that the 24-item MTE scale has construct validity.

\section{Structural Equation Model}

Two separate samples were aggregated to test the structural equation model (SEM). The SEM included not only the measures of the MTE but also five proposed potential consequences: recollection, vividness, fit into life, future behavior, and loyalty behavior. Following the procedure suggested by Anderson and Gerbing (1988), the overall measurement of quality was first confirmed and then the structural model was tested. After confirming the appropriateness of the measurement model, the structural model was examined. For the overall model, the estimated model provided a good fit based on the model fit indices $\left(X^{2} / d f=1.77, X^{2}(\mathrm{df}=769)\right.$ $=1363.04(p<0.001), \mathrm{CFI}=0.98, \mathrm{NNFI}=0.98$, $I F I=0.98$, and RMSEA=0.04). The study results showed that MTE positively influenced a person's ability to recall past tourist experiences $(B=0.31, t=5.24, p<0.001)$ and to do so vividly ( $\beta=0.33, t=5.73, p<0.001$ ). Moreover, the MTE affected a person's belief that the experience was real and not an artifact of general knowledge $(B=0.41, t=$ $6.89, p<0.001)$. MTE had significant positive effects on future behavioural intentions ( $B=$ 0.50, $t=8.63, p<0.001)$. Also, the causal path between MTE and loyalty behavior toward a brand of the company that respondents encountered during a person's experience as a tourist is statistically significant $(~ B=0.20, t=3.71, p<0.001)$.

\section{Theoretical conclusions}

The MTE model suggests that it is composed of seven experiential factors: hedonism, meaningfulness, novelty, knowledge, involvement, local culture, and refreshment. This study also found that MTE influenced the consequent variables, including behavioural intentions and autobiographical memory. The comparison of the standardized path coefficients between the exogenous and endogenous variables showed that MTE most significantly influenced behavioural intentions, such as the intention to revisit and partake in previous tourist activities as well positive word-of-mouth (WOM). This result parallels previous research studies that have shown that memorable experiences contribute to the sales revenue of the provider of the services (Kozak, 2001; Lehto et al., 2004; Schmitt, 1999; Wirtz et al., 2003).

\section{Practical application of the dissertation}

This information provides implications for determining how managers of tourism businesses should prioritize their business resources in developing tourism programs. Tourism programs, as well as the setting in which on-site experiences occur, should be thoroughly evaluated to determine whether they satisfy each of the identified MTE components. After appraising their tourism programs by conducting internal audits and reviews based on the identified MTE dimensions, they can possibly thin out irrelevant or less important program for delivering memorable experiences.

The marketing implications of the results can also be understood by examining the differences in the groups and the magnitude of the standardized path of the coefficients in both the measurement and structural equation models. The findings of the differences in regard to the transportation 
modes used suggest that individuals who use public transportation are more likely to experience local culture and perceive the experience as memorable. Moreover, individuals who stayed in a hostel were more likely to interact with different groups of tourists and experience local culture. Thus, they were likely to experience more novelty and local culture than the other respondents who stayed in different accommodations. In addition, individuals who camped during their travel experience experienced more of a feeling of refreshment than others who used different types of accommodations. Therefore, when the above findings are combined, destination businesses can provide MTE to their visitors by diversifying their accommodations and encouraging the use of public transportation in the course of tourism program.

\section{Content of the dissertation}

\section{Abstract of chapter one}

This study aimed to construct a valid and reliable scale to measure MTE. The scale relies on the following contributory factors: a) hedonism, b) meaningfulness, c) involvement, d) local culture, e) knowledge, f) novelty, and h) refreshment.

\section{Abstract of chapter two}

As a preliminary stage of developing a valid research instrument, an extensive literature review was conducted to identify contributory factors to MTE in order to derive a meaningful conceptual model. As a result, eighteen construct domains (relaxing, hedonic, stimulating, happy, freeing, refreshing, adverse feelings, sociable, meaningfulness, exploration / learning, challenge, value, service, unexpected happenings, personal relevance, novelty-familiarity, activity, and planning) were emerged from the literature review.

\begin{abstract}
of chapter three
Data were collected from U.S. college students at a large Midwestern university. Of the 562 surveys collected, the researcher retained 500 usable responses based on the data screening of missing values and systematic response patterns. Following the scale development procedure suggested by Churchill (1979) and Hinkin (1995), the MTE scale was developed.
\end{abstract}

\section{Abstract of chapter four}

Evidence of construct validity and discriminate validity of the MTE scale were provided by the results of EFA and CFA. The findings of the CFA were cross-validated by splitting the total sample into two 250-case sub-samples. In supporting a consistent factor structure, both calibration and validation samples yielded identical results. All major goodness-of-fit indices indicated the model's good fit to both datasets. Structural relationships between the MTE and consequent variables were tested. The findings indicated a good fit of model to the data.

\section{Abstract of chapter five}

Despite several limitations, the following conclusions are drawn:

1. The 24-item MTE scale developed in this study yielded an appropriate level of reliability and validity.

2. MTE significantly influence future intentions, such as the intention to revisit, partake in the same activities, and WOM.

3. The memorable tourist experience model developed in this study can be used as a framework for conceptualizing MTE and developing tourism programs. 